\title{
Development and Internal Validation of a Practical Model to Identify "Observe" Patients of the European Society of Cardiology 0/1-h Algorithm at Low Risk of a Coronary Diagnosis
}

\author{
Murat Arslan ${ }^{a, b}$ Eric Boersma ${ }^{a}$ Admir Dedic $^{a, b}$ Eric A. Dubois ${ }^{a, c}$ \\ aDepartment of Cardiology, Erasmus Medical Centre, Rotterdam, The Netherlands; ${ }^{b}$ Department of Radiology and \\ Nuclear Medicine, Erasmus Medical Centre, Rotterdam, The Netherlands; 'Department of Intensive Care, Erasmus \\ University Medical Centre, Rotterdam, The Netherlands
}

\section{Keywords}

Non-ST-elevation acute coronary syndrome · Acute chest pain · Observe group · Risk score

\begin{abstract}
Background: Patients with suspected non-ST-elevation acute coronary syndrome (NSTE-ACS) assigned to the "observe" zone of the European Society of Cardiology (ESC) 0/1$\mathrm{h}$ algorithm form a heterogeneous group known to have an unfavourable prognosis. We aim to elucidate the clinical characteristics and management of these patients and generate a model that is predictive of a coronary diagnosis at index visit to the emergency department (ED). Methods: A retrospective observational cohort study, including adult patients presenting to the ED with suspected NSTE-ACS assigned to the "observe" zone of the ESC 0/1-h algorithm. Multivariable logistic regression analysis was performed for the prediction of a coronary diagnosis. Internal validation was performed using bootstrap resampling. Results: A total of 750 patients were included; mean age $66 \pm 13$ years, $35 \%$ women, $50 \%$ with prior history of coronary artery disease (CAD). In 372 (50\%) patients a diagnosis was established within 30 days of index presentation, of whom 169 (45\%) patients had a coronary-related event. Multivariable logistic
\end{abstract}

Karger@karger.com www.karger.com/crd

Karger"

BOPEN ACCESS
(C) 2022 The Author(s)

Published by S. Karger AG, Basel

This article is licensed under the Creative Commons Attribution 4.0 International License (CC BY) (http://www.karger.com/Services/ OpenAccessLicense). Usage, derivative works and distribution are permitted provided that proper credit is given to the author and the original publisher. regression analysis generated a 12-point risk score incorporating 5 variables for the prediction of such event, including type of angina, chest pain occurring during inspiration, prior history of CAD, ST-segment deviation on electrocardiogram, and estimated glomerular filtration rate $<60$. The final model had an optimism-corrected c-statistic of 0.78 ( $95 \%$ confidence interval [CI]: $0.74-0.82$ ). A score $<6$ ruled out a coronary event in $276(37 \%)$ patients, with a sensitivity and negative predictive value of $90 \%$ (95\% Cl: 84-94) and 94\% (91-96), respectively. Conclusion: A score $<6$ identifies patients at low risk of a coronary diagnosis and can guide clinical decision-making in choosing the appropriate diagnostic test.

\footnotetext{
(c) 2022 The Author(s).

Published by S. Karger AG, Basel
}

\section{Introduction}

The introduction of high-sensitivity cardiac troponins (hs-cTn) has led to accelerated diagnostic protocols for the assessment of suspected non-ST-elevation acute coronary syndrome (NSTE-ACS) [1-3]. In the recent European Society of Cardiology (ESC) guideline on the management of patients with a suspected ACS without persistent ST-segment elevation, the use of a 0/1-h algorithm in 
centres with hs-cTn assays is recommended [4]. The ESC 0/1-h algorithm has two separate thresholds for "ruleout" and "rule-in" to optimize diagnostic accuracy and efficacy on both sides of the spectrum.

However, a substantial number of patients do not qualify for either category after repeated hs-cTn testing and are assigned to the "observe" zone. These patients are known to have an unfavourable prognosis, with 1-year survival rates comparable to patients assigned to the "rule-in" category $[5,6]$. Management of these patients remains challenging, and hospital admission and additional (non)invasive testing are often necessary. A more individual approach is recommended where management decisions should be based on the degree of clinical suspicion of NSTE-ACS on a case by case basis [4]. The aim of this study was to elucidate the clinical characteristics and management of patients assigned to the "observe" zone and to generate a model that is predictive of a coronary diagnosis.

\section{Methods}

This retrospective observational cohort study included 750 consecutive adult patients with suspected NSTE-ACS and acute chest pain as principal symptom. Patients presented to the emergency department (ED) at the Erasmus MC, University Medical Centre, Rotterdam, an academic tertiary referral hospital, between February 1, 2012, and January 31,2020. Patients were included if they were aged 18 years and older and assigned to the "observe" zone of the ESC 0/1-h algorithm based on repeated hs-cTn measurements. The start date of February 1, 2012, was chosen as this corresponded with the introduction of the fifth-generation Elecsys hs-cTnT (hs$\mathrm{TnT}$ ) assay (Roche Diagnostics) in our centre. Although the 0/1-h algorithm was first recommended in the 2015 ESC guideline on the management of patients with a suspected ACS without persistent ST-segment elevation, we have also included patients that presented at the ED prior to this recommendation, with repeated hs-cTn measurements which would assign them to the "observe" zone based on current guidelines. Data regarding clinical characteristics, symptoms and signs, electrocardiogram (ECG) data, laboratory results, downstream testing, patient management, and diagnosis were obtained from electronic patient records. This study was conducted according to the principles of the Declaration of Helsinki and was approved by the local institutional review board.

\section{Diagnostic Procedure}

Patients were categorized into three diagnostic groups if the diagnosis was established by treating physicians within 30 days of presentation to the ED: (1) coronary (including NSTE-ACS, chronic coronary syndromes, spontaneous coronary artery dissection, and coronary vasospasm); (2) cardiac, non-coronary (including nonischemic cardiomyopathies, valvular heart disease, brady- and tachyarrhythmias, and [peri] myocarditis); (3) non-cardiac (containing all relevant non-cardiac diagnosis such as pulmonary embolism, anaemia, infections, malignancies, and malignant hypertension).

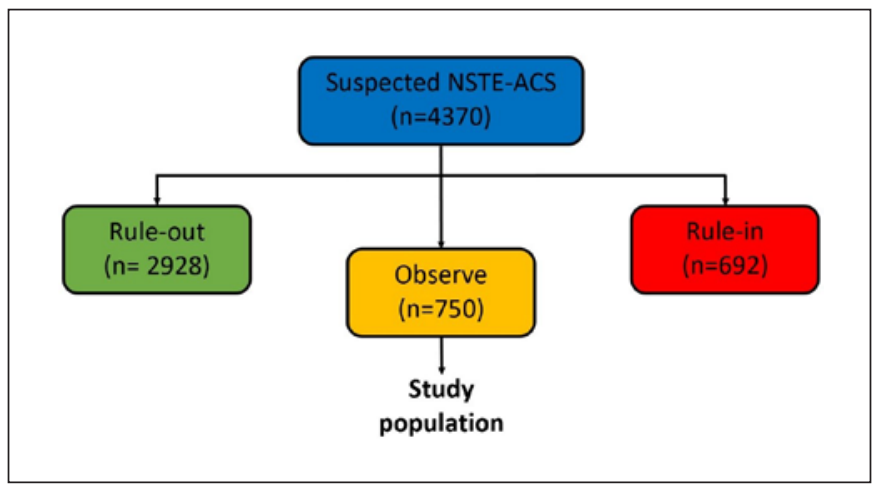

Fig. 1. Flow chart of the study population. NSTE-ACS, non-STelevation acute coronary syndrome.

\section{Study Endpoint}

The primary outcome was a coronary diagnosis at index visit to the ED, established by the treating physician, based on all available clinical data.

\section{Statistical Analysis}

Continuous data are presented as mean $\pm \mathrm{SD}$ or median (interquartile ranges), and categorical data are presented as proportions (percentages). We used logistic regression analysis for the prediction of a coronary diagnosis. Initially, univariable analysis was performed to evaluate the ability of chosen variables to predict a coronary diagnosis. We examined 29 potential predictors, including sex, age, cardiovascular risk factors, medication, symptoms, ECG findings, and laboratory results. Variables with a $p<0.10$ entered the multivariate stage, and a multivariable logistic regression model was constructed to predict a coronary diagnosis, using the stepwise backward selection method, with a value of $p=0.05$ as a model-entry criterion. Variables were checked for multicollinearity using correlations and variance inflation factors to avoid redundancy in the prediction model. Dichotomization of the continuous variable estimated glomerular filtration rate (eGFR) was based on the clinically relevant threshold for kidney disease. The relative magnitude of the model regression coefficients from statistically significant variables in the final multivariable model was used to calculate an individual patient's risk score for the prediction of a coronary diagnosis. Each point assigned to covariates in the final multivariable model was rounded to the nearest integer to simplify the calculation of the final score in clinical practice. The model discrimination abilities were evaluated by the c-statistic of the final multivariate model. For further internal validation, 1,000 bootstrap samples were obtained, and an optimism-corrected cstatistic was calculated [7]. The optimal cut-off value for the risk score was chosen after visual inspection of the diagnostic accuracy parameters at various cut-offs, with the aim to optimize the efficacy (defined as the number of patients ruled out) of the score while maintaining high sensitivity and negative predictive value (NPV) for the prediction of a coronary diagnosis. Receiver-operating characteristic curve analysis of the computed risk score was compared with a validated risk score, namely the History, ECG, Age, Risk factors, and Troponin (HEART) score $[8,9]$. 
Table 1. Baseline characteristics of patients assigned to the "observe zone" of the ESC 0/1-h algorithm

\begin{tabular}{|c|c|c|c|c|}
\hline & $\begin{array}{l}\text { Study cohort } \\
(N=750)\end{array}$ & $\begin{array}{l}\text { Cardiac, coronary } \\
(N=169)\end{array}$ & $\begin{array}{l}\text { Rest of the cohort } \\
(N=581)\end{array}$ & $p$ value \\
\hline Age, years & $66 \pm 13$ & $66 \pm 12$ & $65 \pm 13$ & 0.33 \\
\hline \multicolumn{5}{|l|}{ Risk factors } \\
\hline Active smoker & $126(16.8)$ & $28(16.6)$ & $98(16.9)$ & 0.93 \\
\hline Hypertension & $479(63.9)$ & $121(71.6)$ & $358(62.0)$ & 0.02 \\
\hline Diabetes mellitus & $227(30.3)$ & $59(34.9)$ & $168(29.0)$ & 0.14 \\
\hline Family history for CAD & $188(25.1)$ & $53(31.4)$ & $135(23.2)$ & 0.03 \\
\hline PAD & $65(8.7)$ & $11(6.5)$ & $54(9.3)$ & 0.28 \\
\hline Prior stroke/TIA & $105(14.0)$ & $20(11.8)$ & $85(14.6)$ & 0.36 \\
\hline Prior history of CAD & $375(50.0)$ & $120(71.0)$ & $255(43.9)$ & $<0.0001$ \\
\hline \multicolumn{5}{|l|}{ Medication } \\
\hline Statin & $449(59.9)$ & $115(68.0)$ & $334(57.5)$ & 0.02 \\
\hline Beta blocker & $414(55.2)$ & $99(58.5)$ & $315(54.2)$ & 0.33 \\
\hline Diuretics & $247(32.9)$ & $47(27.8)$ & $200(0.34)$ & 0.11 \\
\hline $\mathrm{CCB}$ & $181(24.1)$ & $55(32.5)$ & $126(21.7)$ & 0.004 \\
\hline \multicolumn{5}{|l|}{ Symptoms and signs } \\
\hline \multicolumn{5}{|l|}{ Chest pain* } \\
\hline Typical angina & $122(16.3)$ & $61(36.1)$ & $61(10.5)$ & $<0.0001$ \\
\hline Atypical angina & $346(46.1)$ & $82(48.5)$ & $264(45.4)$ & \\
\hline Non-anginal chest pain & $282(37.6)$ & $26(15.4)$ & $256(44.1)$ & \\
\hline Chest pain occurring with inspiration & $51(6.8)$ & $2(1.2)$ & $49(8.4)$ & 0.001 \\
\hline \multicolumn{5}{|l|}{ Blood pressure, $\mathrm{mm} \mathrm{Hg}$} \\
\hline Systolic & $145.5 \pm 42.0$ & $145.7 \pm 23.2$ & $144.1 \pm 26.4$ & 0.48 \\
\hline \multicolumn{5}{|l|}{ Laboratory } \\
\hline \multicolumn{5}{|c|}{ Time from chest pain onset to first blood draw } \\
\hline$<3 \mathrm{~h}$ & $238(31.7)$ & $51(30.2)$ & $187(32.2)$ & 0.23 \\
\hline$>3 \mathrm{~h}$ & $337(50.3)$ & $71(42.0)$ & $266(45.8)$ & \\
\hline$>24 \mathrm{~h}$ & $155(20.7)$ & $44(26.0)$ & $111(19.1)$ & \\
\hline eGFR & $69 \pm 22.8$ & $72 \pm 21$ & $68 \pm 23$ & 0.04 \\
\hline \multicolumn{5}{|l|}{ Risk scores } \\
\hline HEART score & $5.3 \pm 1.6$ & $6.1 \pm 1.6$ & $5.1 \pm 1.5$ & 0.0001 \\
\hline TIMI score & $2.9 \pm 1.5$ & $3.6 \pm 1.5$ & $2.7 \pm 1.4$ & 0.0001 \\
\hline GRACE score & $118 \pm 29.7$ & $119 \pm 29.3$ & $118 \pm 29.8$ & 0.66 \\
\hline \multicolumn{5}{|c|}{$\begin{array}{l}\text { Patients with ECGs suggestive of an ST-elevation myocardial infarction were excluded from this analysis. ACE, angiotensin-converting- } \\
\text { enzyme; ARB, angiotensin II receptor blocker; CAD, coronary artery disease; CCB, calcium channel blocker; DOAC, direct oral anticoagulant; } \\
\text { ECG, electrocardiogram; eGFR, estimated glomerular filtration rate; ESC, European Society of Cardiology; GRACE, The Global Registry of } \\
\text { Acute Coronary Events; HEART, history; EKG, age, risk factors, and troponin; PAD, peripheral arterial disease; TIA, transient ischemic stroke; } \\
\text { TIMI, the thrombolysis in myocardial infarction; VKA, vitamin K antagonist. *Categorized according to the Diamond and Forrester } \\
\text { classification [10]." ST-segment deviation was defined as } \geq 1 \mathrm{~mm} \text { ST-segment deviation in one or more leads [11]. }\end{array}$} \\
\hline
\end{tabular}

A Score to Identify “Observe" Patients with a Coronary Diagnosis
Cardiology 2022;147:251-260 DOI: $10.1159 / 000523718$ 
Table 2. Use of diagnostic modalities and management of patients assigned to the "observe zone" of the ESC 0/1$\mathrm{h}$ algorithm within 30 days of index presentation

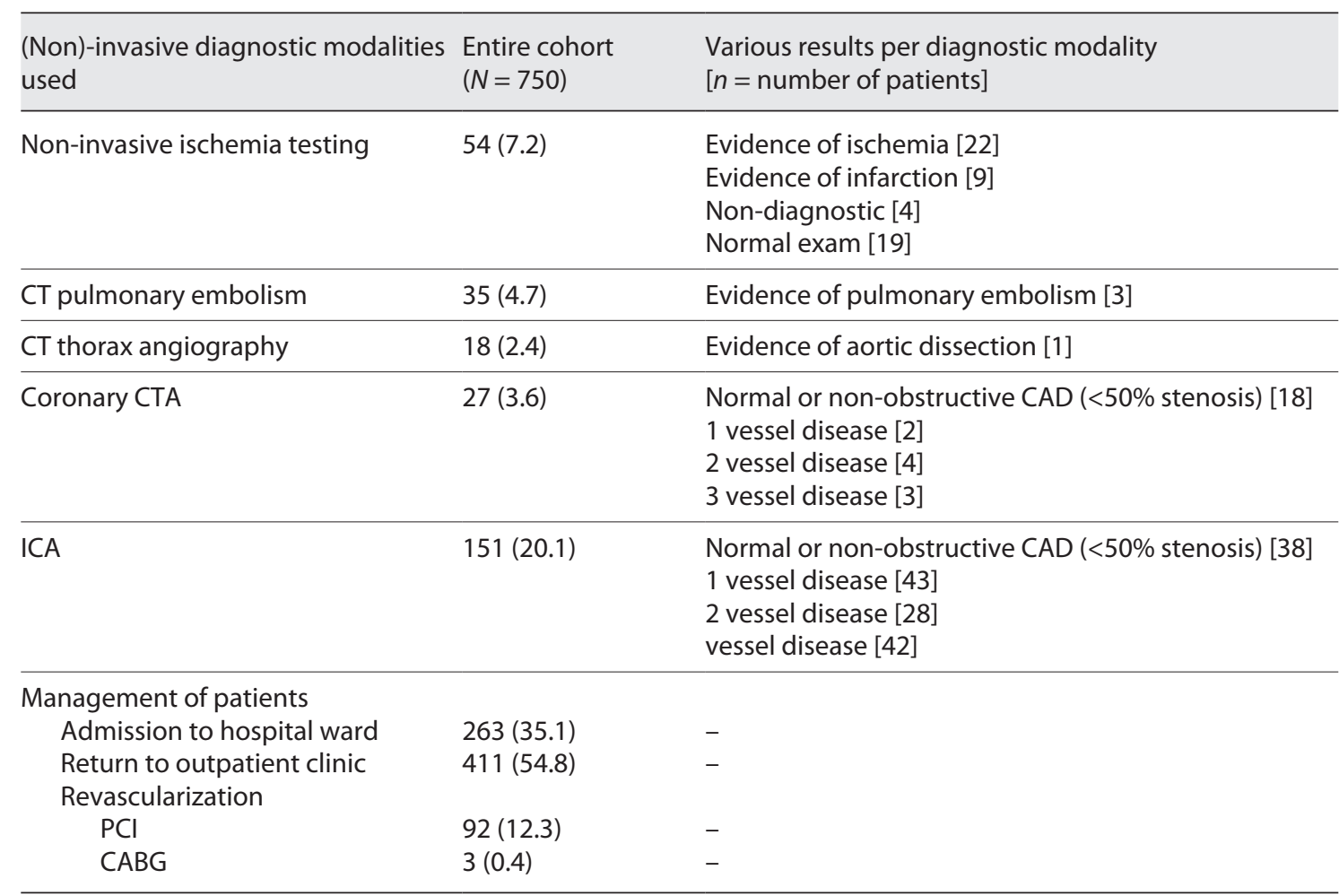

Non-invasive ischemia testing included: Stress ECG, stress echocardiography; SPECT, and cardiac MRI, CABG, coronary artery bypass grafting; $C A D$, coronary artery disease; CCTA, coronary computed tomography angiography; ESC, European Society of Cardiology; ICA, invasive coronary angiography; $\mathrm{PCl}$, percutaneous coronary intervention.

Survival data of all patients were acquired using in-hospital medical records and municipal civil registry. Cumulative survival of up to 1-year follow-up was calculated and stratified by diagnostic categories, i.e., (1) coronary; (2) cardiac, non-coronary, and (3) non-cardiac, with corresponding 95\% confidence intervals and plotted using Kaplan-Meier curves. The log-rank test was used to examine differences between diagnostic categories in the KaplanMeier analyses, correcting for multiple comparisons using the Bonferroni method.

All statistical analyses were performed using SPSS version 24.0 (IBM, Armonk, NY, USA) and R version 4.0.2 (R Project for statistical computing, Vienna, Austria). All tests were two-tailed, and a $p$ value $<0.05$ was considered statistically significant.

\section{Results}

Between February 1, 2012, and January 31, 2020, 4,370 consecutive adult patients presented with suspected NSTE-ACS and acute chest pain, of whom 2,928 patients were triaged towards "rule-out" and 692 patients as "rule- in" by the ESC 0/1-h algorithm. In total, 750 suspected NSTE-ACS patients were assigned to the "observe zone" of the ESC 0/1-h algorithm and comprised the study population (Fig. 1). Baseline characteristics of included patients are shown in Table 1 . The mean age was $66 \pm 13$ years and the proportion of women was 35\%. Half of the patients had a prior history of coronary artery disease (CAD). In $32 \%$ of the patients first blood draw was performed within $3 \mathrm{~h}$ of chest pain onset. The median hsTnT value in the study cohort at baseline was 18 (14-25) and the second median hs-TnT value was also 18 (14-25). Patients with a coronary diagnosis were more often male with pre-existing CAD and more often had ischemic ECG abnormalities as compared to the rest of the cohort (Table 1).

\section{Resource Use in the Observe Group}

Non-invasive ischemia testing was performed in 54 (7\%) patients within 30 days of index presentation and 


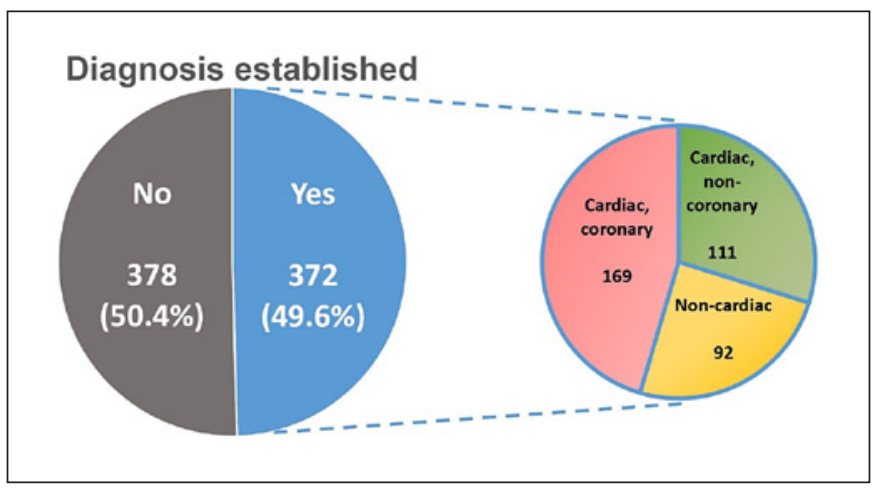

Fig. 2. Figure depicting the proportion of patients with an established diagnosis related to index visit, based on all available clinical data with their corresponding diagnostic categories.

revealed signs of myocardial ischemia or infarction in 31 patients (Table 2). Invasive coronary angiography (ICA) was performed in 151 (20\%) patients, of whom $92(12 \%)$ patients underwent percutaneous coronary intervention and $3(0.4 \%)$ patients underwent coronary artery bypass grafting within 30 days of index presentation. 263 (35\%) patients were admitted to the hospital after evaluation at the ED and 411 (55\%) patients were sent home with an appointment at the outpatient clinic (Table 2).

\section{Final Diagnosis}

In $372(50 \%)$ patients, a clinically relevant diagnosis related to index visit was established, based on all available clinical data (Fig. 2). The remaining 378 (50\%) patients were discharged from the ED after exclusion of acute significant pathologies and had no obvious diagnosis after 30 days that explained their chest pain. Among the patients with an established diagnosis, the most common diagnosis was coronary disease, followed by "cardiac, non-coronary" diseases and finally non-cardiac diseases.

\section{Prediction of a Coronary Diagnosis}

Univariable regression analysis of the relationship with a coronary diagnosis identified an association with female sex, hypertension, prior history of CAD, aspirin use, statin use, P2Y12 inhibitor use, calcium channel blocker use (a)typical angina, chest pain occurring during inspiration, heart rate, ST-segment deviation, and pathological Q waves on ECG and eGFR $<60$ (Table 3). Of these, prior history of CAD, (a)typical angina, chest pain occurring during inspiration, ST-segment deviation on ECG, and eGFR $<60$ remained significant independent predictors of a coronary diagnosis in the multivariable analysis. The final multivariate model had a $c$-statistic of 0.78 (0.74-0.82). Bootstrap internal validation revealed an optimism-corrected c-statistic of 0.77 (0.73-0.81).

Points assigned to the five covariates in the final multivariable model are depicted in Table 4. From the final multivariable model, a 5-item risk score was generated with a score from 0 to 12 . Prior history, ECG, kidney function, and type of chest pain (PEKT) score is composed of prior history of CAD ( 2 points), acute chest pain: atypical angina (2 points) or typical angina (4 points), chest pain occurring during inspiration ( -3 points), STsegment deviation ( 2 points), and eGFR $<60$ ( -1 point), with all patients receiving an additional 4 points regardless of their symptoms at presentation. The median score in the study cohort was 6 [5-8]. Sensitivity, specificity, positive predictive value, and NPV according to the PEKT score risk score are presented in Table 5. Bootstrap internal validation of the PEKT score revealed an optimismcorrected c-statistic of $0.78(0.74-0.82)$. A PEKT score lower than 6 identified 276 (37\%) patients at low risk of having a coronary diagnosis, with a sensitivity and NPV of 89.9 (84.4-94.0) and 93.8 (90.6-96.0), respectively. In comparison, the HEART score had a $c$-statistic of 0.69 (0.64-0.74) (Fig. 3).

\section{Survival}

The overall 30-day and 1-year cumulative survival in patients assigned to the "observe zone" were 0.98 (0.97$0.99)$ and $0.92(0.90-0.94)$, respectively. The 30-day cumulative survival in patients with "no diagnosis," "coronary," "cardiac, non-coronary," and "non-cardiac" diagnoses were 0.98 (0.97-0.99), 0.99 (0.98-1.00), 0.99 (0.97-1.00), and 0.97 (0.93-1.00), respectively. The 1-year cumulative survival in patients with "no diagnosis," "coronary," "cardiac, non-coronary," and "non-cardiac" diagnoses were 0.92 (0.89-0.95), 0.96 (0.94-0.99), 0.91 (0.86-0.96), and 0.88 (0.81-0.95), respectively. Figure 4 shows the Kaplan-Meier plots stratified by diagnostic categories. Pairwise log-rank tests revealed a significant difference in 1-year cumulative survival between the cardiac, coronary group as compared to the non-cardiac group $(p=0.007, a=0.008)$.

\section{Discussion}

In this retrospective observational study, we investigated the clinical characteristics and management of suspected NSTE-ACS patients assigned to the "observe" 
Table 3. Univariable analysis and adjusted multivariable analysis for the prediction of a coronary diagnosis

\begin{tabular}{|c|c|c|c|c|}
\hline Covariate & $\begin{array}{l}\text { Univariable analysis } \\
\text { OR }(95 \% \mathrm{Cl})\end{array}$ & $p$ value & $\begin{array}{l}\text { Multivariable analysis } \\
\text { OR }(95 \% \mathrm{Cl})\end{array}$ & $p$ value \\
\hline Female sex & $0.58(0.39-0.85)$ & 0.005 & - & - \\
\hline Age (per 1-year increase) & $1.007(0.99-1.02)$ & 0.32 & - & - \\
\hline \multicolumn{5}{|l|}{ Risk factors } \\
\hline Smoking & $0.94(0.59-1.49)$ & 0.80 & - & \multirow[t]{7}{*}{-} \\
\hline Hypertension & $1.54(1.06-2.24)$ & 0.023 & - & \\
\hline Dyslipidemia & $1.32(0.98-1.76)$ & 0.067 & - & \\
\hline Diabetes mellitus & $1.31(0.91-1.89)$ & 0.14 & - & \\
\hline Family history for CAD & $1.42(0.98-2.05)$ & 0.064 & - & \\
\hline PAD & $0.68(0.35-1.33)$ & 0.26 & - & \\
\hline Prior stroke/TIA & $0.78(0.47-1.32)$ & 0.36 & - & \\
\hline Prior history of CAD & $3.1(2.16-4.54)$ & $<0.0001$ & $2.64(1.75-3.98)$ & $<0.0001$ \\
\hline \multicolumn{5}{|l|}{ Medication } \\
\hline Aspirin & $2.02(1.43-2.85)$ & $<0.0001$ & - & - \\
\hline Oral anticoagulation & $0.86(0.59-1.27)$ & 0.45 & - & - \\
\hline ACE inhibitor or ARB & $1.14(0.81-1.61)$ & 0.45 & - & - \\
\hline P2Y12 inhibitor & $1.47(0.99-2.18)$ & 0.06 & - & - \\
\hline Statin & $1.61(1.11-2.31)$ & 0.011 & - & - \\
\hline Beta blocker & $1.21(0.86-1.72)$ & 0.28 & - & - \\
\hline Diuretics & $0.73(0.50-1.07)$ & 0.11 & - & - \\
\hline $\mathrm{CCB}$ & $1.74(1.19-2.54)$ & 0.004 & - & - \\
\hline \multicolumn{5}{|l|}{ Symptoms } \\
\hline \multicolumn{5}{|l|}{ Chest pain* } \\
\hline Non-anginal & 1 (reference) & - & - & - \\
\hline Atypical angina & $3.06(1.91-4.91)$ & $<0.0001$ & $3.06(1.84-5.09)$ & $<0.0001$ \\
\hline Typical angina & $9.85(5.75-16.85)$ & $<0.0001$ & $8.55(4.77-15.33)$ & $<0.0001$ \\
\hline Chest pain occurring during inspiration & $0.130(0.03-0.54)$ & 0.005 & $0.18(0.042-0.80)$ & 0.02 \\
\hline \multicolumn{5}{|c|}{ ECG } \\
\hline $\mathrm{AF}$ & $1.49(0.91-2.45)$ & 0.11 & - & - \\
\hline Heart rate (per min) & $0.99(0.98-0.999)$ & 0.03 & - & - \\
\hline Left ventricular hypertrophy & $0.49(0.19-1.27)$ & 0.14 & - & - \\
\hline ST-segment deviation ${ }^{\#}$ & $2.81(1.76-4.49)$ & $<0.0001$ & $3.00(1.75-5.14)$ & $<0.0001$ \\
\hline T Wave inversion & $1.19(0.81-1.75)$ & 0.39 & - & - \\
\hline Pathological Q waves & $1.80(1.08-2.98)$ & 0.023 & - & - \\
\hline \multicolumn{5}{|l|}{ Laboratory results } \\
\hline eGFR $<60$ & $0.71(0.49-1.03)$ & 0.07 & $0.56(0.37-0.86)$ & 0.008 \\
\hline \multicolumn{5}{|c|}{$\begin{array}{l}\text { Patients with ECGs suggestive of an ST-elevation myocardial infarction were excluded from this analysis. ACE, } \\
\text { angiotensin-converting-enzyme; AF, atrial fibrillation or atrial flutter; ARB, angiotensin II receptor blocker; CAD, } \\
\text { coronary artery disease; CCB, calcium channel blocker; DOAC, direct oral anticoagulant; ECG, electrocardiogram; } \\
\text { eGFR, estimated glomerular filtration rate; ESC, European Society of Cardiology; OR, odds ratio; PAD, peripheral } \\
\text { arterial disease; TIA, transient ischemic stroke; VKA, vitamin K antagonist. }{ }^{*} \text { Categorized according to the Diamond } \\
\text { and Forrester classification [10]. }{ }^{\text {ST }} \text {-segment deviation was defined as } \geq 1 \mathrm{~mm} \text { ST-segment deviation in one or more } \\
\text { leads [11]. }\end{array}$} \\
\hline
\end{tabular}

zone of the ESC 0/1-h algorithm and we developed and internally validated the PEKT score, a simple 5-item tool to identify patients at low risk of a coronary diagnosis. We hereby report several important findings.

First, "observe" patients were generally elderly men with a prior history of CAD. Second, a clinically relevant diagnosis related to index visit at the ED was established in only
$50 \%$, with CAD being the most commonly established diagnosis. Third, cumulative 1-year survival in "observe" patients was $92 \%$ and patients with a coronary diagnosis appeared to have a relatively better survival outcome as compared to patients in other diagnostic categories.

Previous data from the Advantageous Predictors of Acute Coronary Syndromes Evaluation group portrayed 
Table 4. Individual variable scores of the multivariable model for the prediction of a coronary diagnosis

\begin{tabular}{llll}
\hline Variables & OR $(95 \% \mathrm{Cl})$ & Beta coefficient & Score \\
\hline Prior history of CAD & $2.65(1.75-4.03)$ & 0.969 & 2 \\
Chest pain* & & & 0 \\
$\quad$ Non-anginal & 1 (reference) & - & 2 \\
$\quad$ Atypical angina & $2.62(1.58-4.37)$ & 1.117 & 4 \\
$\quad$ Typical angina & $8.44(4.69-15.17)$ & 2.146 & -3 \\
Chest pain occurring during inspiration & $0.18(0.042-0.79)$ & -1.702 & 2 \\
ST-segment deviation\# & $2.83(1.64-4.87)$ & 1.098 & -1 \\
eGFR $<60$ & $0.61(0.40-0.94)$ & -0.578 & \\
\hline
\end{tabular}

In the final score, all patients receive an additional 4 points regardless of their symptoms at presentation. Patients with ECGs suggestive of an ST-elevation myocardial infarction were excluded from this analysis. CAD, coronary artery disease; CCB, calcium channel blocker; eGFR, estimated glomerular filtration rate; OR, odds ratio. * Categorized according to the Diamond and Forrester classification [10]. "ST-segment deviation was defined as $\geq 1$ $\mathrm{mm}$ ST-segment deviation in one or more leads [11].

Table 5. Predictive value of the "derived" risk score for coronary events at various cut-offs

\begin{tabular}{lllllll}
\hline Criterion & Criterion & Ruled out, $n(\%)$ & Sensitivity $(95 \% \mathrm{Cl})$ & Specificity $(95 \% \mathrm{Cl})$ & $\mathrm{PPV}(95 \% \mathrm{Cl})$ & $\mathrm{NPV}(95 \% \mathrm{Cl})$ \\
\hline$\geq 0$ & $\geq 0$ & $0(0)$ & $100.0(97.8-100.0)$ & $0.0(0.0-0.6)$ & $22.5(22.5-22.5)$ & $\mathrm{NA}$ \\
$>0$ & $<1$ & $3(0.4)$ & $100.0(97.8-100.0)$ & $0.5(0.1-1.5)$ & $22.6(22.5-22.7)$ & 100 \\
$>1$ & $<2$ & $14(1.9)$ & $100.0(97.8-100.0)$ & $2.4(1.3-4.0)$ & $23.0(22.7-23.2)$ & 100 \\
$>2$ & $<3$ & $24(3.2)$ & $100.0(97.8-100.0)$ & $4.1(2.7-6.1)$ & $23.3(23.0-23.6)$ & 100 \\
$>3$ & $<4$ & $75(10.0)$ & $98.8(95.8-99.9)$ & $12.6(10.0-15.5)$ & $24.7(24.1-25.4)$ & $97.3(90.1-99.3)$ \\
$>4$ & $<5$ & $170(22.7)$ & $95.3(90.9-97.9)$ & $27.9(24.3-31.7)$ & $27.8(26.6-29.0)$ & $95.3(91.0-97.6)$ \\
$>5$ & $<6$ & $276(36.8)$ & $89.9(84.4-94.0)$ & $44.6(40.5-48.7)$ & $32.1(30.2-34.0)$ & $93.8(90.6-96.0)$ \\
$>6$ & $<7$ & $438(58.4)$ & $74.0(66.7-80.4)$ & $67.8(63.8-71.6)$ & $40.1(36.6-43.7)$ & $90.0(87.3-92.1)$ \\
$>7$ & $<8$ & $515(68.7)$ & $62.1(54.4-69.5)$ & $77.6(74.0-81.0)$ & $44.7(40.0-49.5)$ & $87.6(85.3-89.6)$ \\
$>8$ & $<9$ & $643(85.7)$ & $37.3(30.0-45.0)$ & $92.4(90.0-94.4)$ & $58.9(50.4-66.9)$ & $83.5(81.8-85.1)$ \\
$>9$ & $<10$ & $680(90.7)$ & $26.6(20.1-34.0)$ & $95.7(93.7-97.2)$ & $64.3(53.2-74.0)$ & $81.8(80.3-83.1)$ \\
$>10$ & $<11$ & $739(98.5)$ & $4.7(2.1-9.1)$ & $99.5(98.5-99.9)$ & $72.7(41.7-90.9)$ & $78.2(77.6-78.8)$ \\
$>11$ & $<12$ & $743(99.1)$ & $3.6(1.3-7.6)$ & $99.8(99.0-100.0)$ & $85.7(42.1-98.0)$ & $78.1(77.6-78.6)$ \\
$>12$ & $<13$ & $750(100)$ & $0.0(0.0-2.2)$ & $100(99.4-100.0)$ & NA & $77.5(77.5-77.5)$ \\
\hline
\end{tabular}

$\mathrm{Cl}$, confidence interval; NA, not applicable; NPV, negative predictive value; PPV, positive predictive value.

similar clinical characteristics of "observe" patients as found in our study cohort, with the population generally being older men and with known pre-existing CAD [6, $12]$. The proportion of men in our study was even higher in patients with a coronary-related diagnosis. Interestingly, in half of the patients, NSTE-ACS or a life-threatening condition was excluded, however, an underlying cause for their symptoms was not found within 30 days of presentation at the ED. Patients without a diagnosis still have an unfavourable prognosis beyond 30 days of presentation, representing a heterogeneous group of patients with complex (non-)cardiac causes of troponin leakage. Pa- tients with a coronary diagnosis seem to have a better outcome when compared to other diagnostic categories. A possible explanation is that patients with a coronary diagnosis had a more readily treatable and simple event as compared to other diagnostic categories. In addition, we cannot rule out that patients without a coronary diagnosis may have had undiagnosed CAD, which untreated may have contributed to the survival differences.

\section{Resource Use}

Our data shows that the treating physician had a preference for ICA as compared to other imaging mo- 
Fig. 3. ROC curves of risk scores for the prediction of a coronary diagnosis. AUC, area under the curve; HEART score, history, ECG, age, risk factors, and troponin score; PEKT score, prior history, ECG, kidney function, and type of chest pain score; ROC, receiver-operating characteristic.

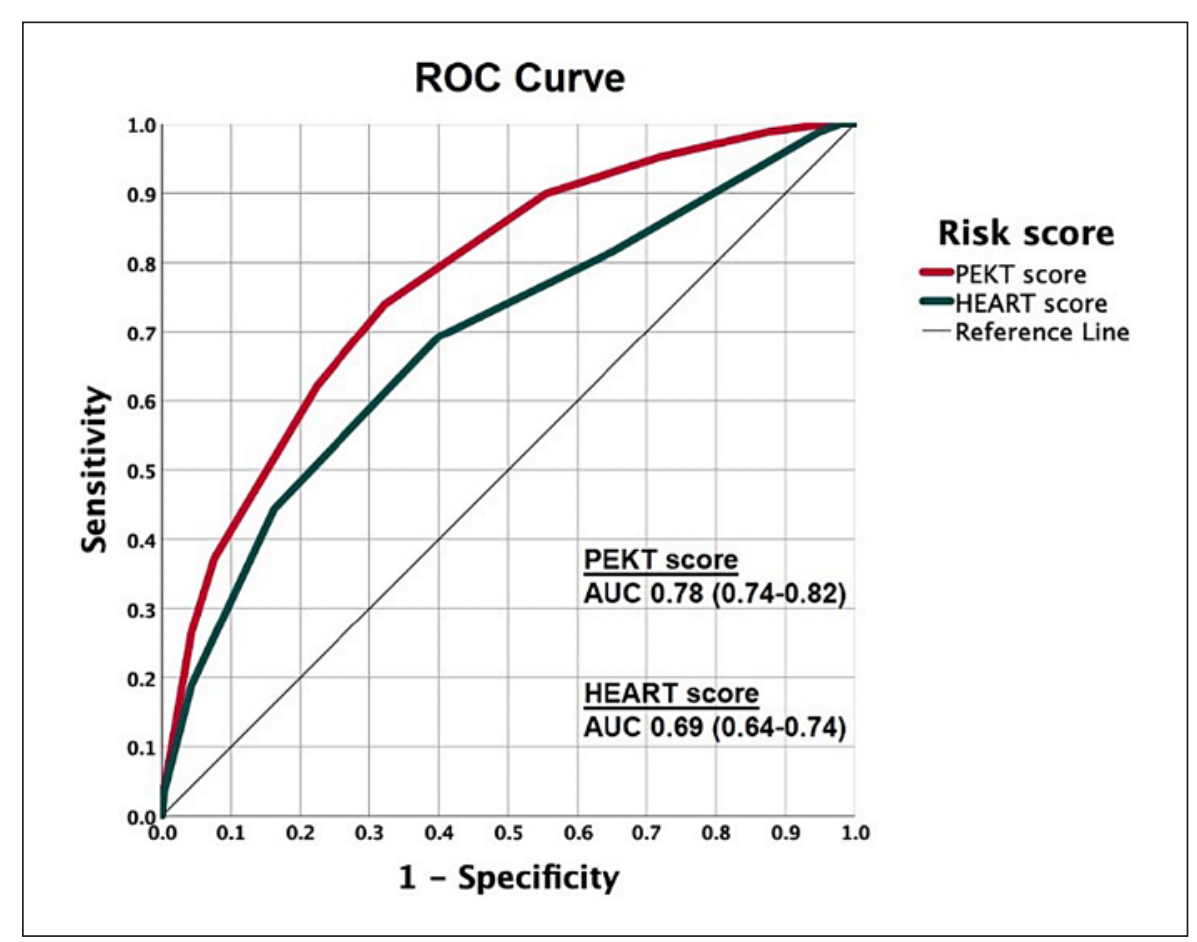

dalities, such as coronary computed tomography angiography (CCTA), in patients assigned to the "observe zone." The reason may be a combination of available expertise built over the years of using ICA and a lack of evidence for the usefulness of CCTA in the "observe zone." Previous trials in low-risk patients with suspected NSTE-ACS have shown the efficacy of CCTA; however, this still remains to be seen for patients assigned to the observe zone $[13,14]$. Currently, we are in the final phase of a prospective, double-blind, observational, multicentre study (COURSE trial) that aims to determine the usefulness of CCTA in patients with lowrange positive troponins [15].

\section{PEKT Score}

Patients in the "observe" group with pre-existing CAD have a more than 2-fold increase in the incidence of a coronary diagnosis when presenting to the ED with acute chest pain. Considering that in our study a large proportion of "observe" patients have pre-existing CAD, this is an important risk factor to take into consideration in further patient management decisions at the ED.

Acute chest pain plays a central role in our novel risk score, with typical angina having the greatest weight in predicting a coronary diagnosis among all 5 predictors. However, patients with chest pain which specifically worsened during inspiration have a clearly lower likeli- hood of having a coronary diagnosis. This highlights the importance of proper history taking in patients with chest pain complaints and its downstream effect on the decision to perform an ICA.

Patients in the "observe" group presenting with impaired renal function defined as eGFR $<60$ were less likely to have a coronary diagnosis as compared to patients with normal renal function. This phenomenon is partially ascribed to non-ischemic elevation of troponin levels in patients with kidney disease, as opposed to myocardial necrosis leading to a rise of troponin levels in patients with myocardial infarction $[16,17]$. In patients with renal dysfunction, diagnostic protocols such as the ESC 0/1-h algorithm, also have a decreased specificity for type 1 myocardial infarction [18-20], i.e., even if patients with renal dysfunction develop myocardial infarction, it is less likely to have a coronary cause.

The PEKT score performed well and was able to discriminate between patients that need further coronary investigation and those that do not. A PEKT score lower than 6 identifies patients at low risk of coronary diagnosis with a relatively high NPV. In these patients treating physicians may consider an alternative diagnosis. It is important to note that our risk score warrants prospective external validation prior to use in daily clinical practice. The HEART score, which has been developed and validated in patients with suspected NSTE-ACS, was a relatively 


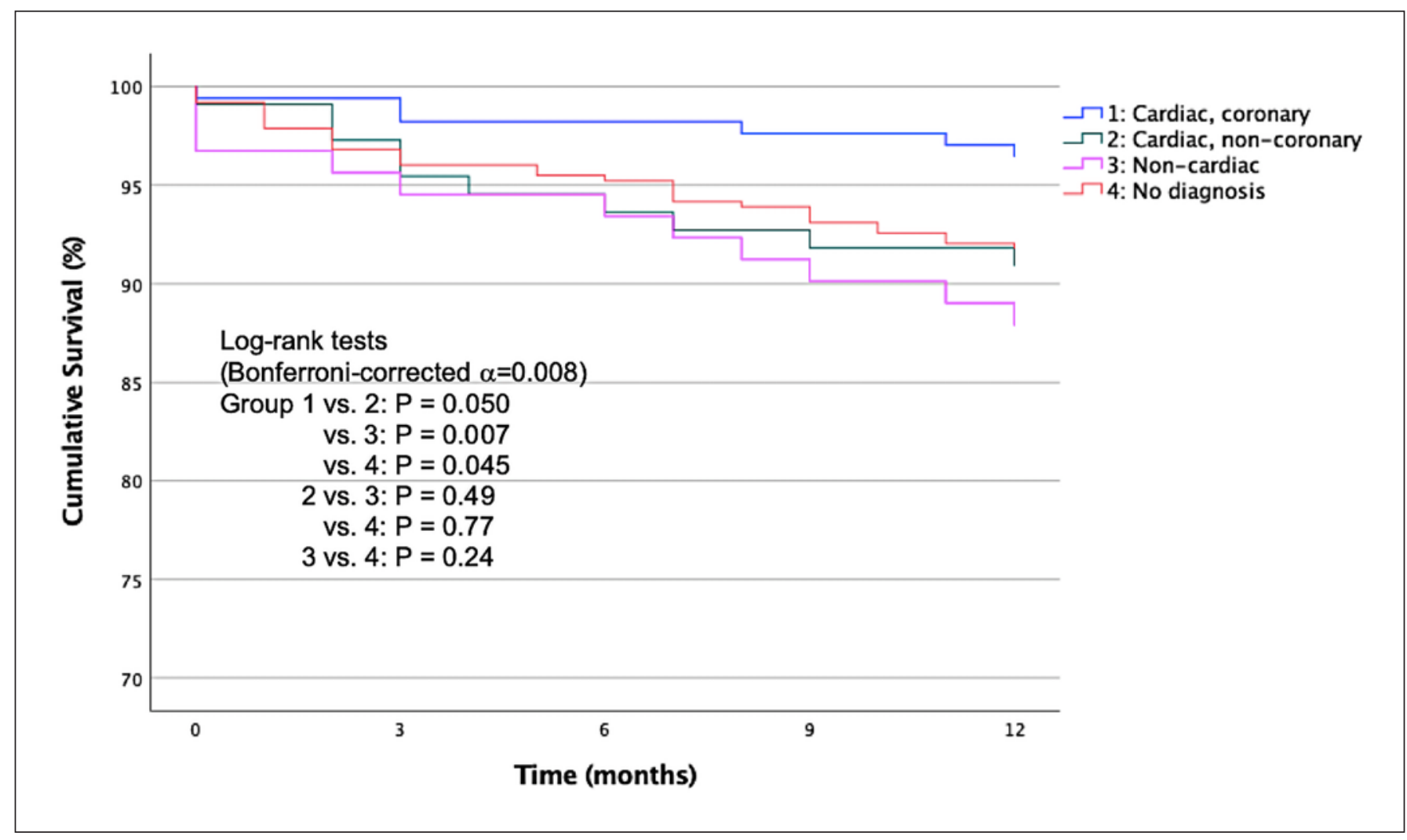

Fig. 4. One-year Kaplan-Meier survival curves in patients assigned to the "observe zone" of the ESC $0 \mathrm{~h} / 1 \mathrm{~h}$ algorithm stratified based on diagnostic categories.

good predictor of a coronary diagnosis in our cohort but did not perform as well.

Our scoring system is simple and composed of easily obtainable clinical characteristics. The PEKT score may provide clinicians at the ED an opportunity to simplify their decision-making and may help reduce variability in patient management for the "observe" group.

\section{Limitations}

The present study has several limitations that should be acknowledged. First, its single-centre, retrospective design has some inherent limitations, such as selection bias. Thus, the study sample may not be a true representation of "observe" patients, which limits generalizability. Second, the final diagnosis was established by treating physicians as opposed to a central adjudication process of final diagnoses. Lack of final diagnosis adjudication in turn may have affected the developed risk score, considering that a potential change in final diagnosis after adjudication would alter the outcome of our diagnosis-based risk score.

A Score to Identify "Observe" Patients with a Coronary Diagnosis

\section{Conclusion}

Patients assigned to the "observe" group were in general elderly men with known pre-existing CAD. A substantial proportion of patients do not have an established diagnosis after 30 days and patients with a coronary diagnosis seem to have a better outcome when compared to other diagnostic categories. A PEKT score $<6$ identifies patients at low risk of a coronary diagnosis and may obviate the need for further coronary investigation in up to $40 \%$ of patients in our cohort.

\section{Statement of Ethics}

This study was conducted according to the principles of the Declaration of Helsinki, approved by the Medical Research Ethics Committee of Erasmus Medical Center in Rotterdam, The Netherlands (registration number MEC-2020-0157) and a waiver of informed consent was obtained. 


\section{Conflict of Interest Statement}

The authors declare that there is no conflict of interest.

\section{Funding Sources}

This work was supported by a grant from Erasmus MC and a research grant from the Erasmus MC Thorax Foundation (project grant B4).

\section{Author Contributions}

Murat Arslan: conceptualization, data curation, formal analysis, project administration, visualization, and writing - original draft. Eric Boersma: formal analysis, methodology, investigation, and writing - review and editing. Admir Dedic: conceptualization, data curation, formal analysis, investigation, methodology, project administration, resources, supervision, visualization, writing original draft, and writing - review and editing. Eric A. Dubois: conceptualization, data curation, formal analysis, funding acquisition, resources, supervision, visualization, writing - original draft, and writing - review and editing.

\section{Data Availability Statement}

All data generated or analysed during this study are included in this article. Further enquiries can be directed to the corresponding author.

\section{References}

1 Chapman Andrew R, Hesse K, Andrews J, Ken Lee K, Anand A, Shah Anoop SV, et al. High-Sensitivity cardiac troponin I and clinical risk scores in patients with suspected acute coronary syndrome. Circulation. 2018; 138(16):1654-65.

2 Mueller C, Giannitsis E, Christ M, OrdóñezLlanos J, deFilippi C, McCord J, et al. Multicenter evaluation of a $0-\mathrm{h} / 1-\mathrm{h}$ algorithm in the diagnosis of myocardial infarction with high-sensitivity cardiac troponin T. Ann Emerg Med. 2016;68(1):76-87.e4.

3 Than M, Cullen L, Aldous S, Parsonage WA, Reid CM, Greenslade J, et al. 2-h accelerated diagnostic protocol to assess patients with chest pain symptoms using contemporary troponins as the only biomarker: the ADAPT trial. J Am Coll Cardiol. 2012;59(23):2091-8.

4 Collet JP, Thiele H, Barbato E, Barthélémy O, Bauersachs J, Bhatt DL, et al. ESC guidelines for the management of acute coronary syndromes in patients presenting without persistent ST-segment elevation. Eur Heart J. 2021; 42(14):1289-367.

5 Chiang $\mathrm{CH}$, Chiang $\mathrm{CH}$, Lee GH, Gi WT, Wu YK, Huang SS, et al. Safety and efficacy of the European society of cardiology $0 / 1-\mathrm{h}$ algorithm for diagnosis of myocardial infarction: systematic review and meta-analysis. Heart. 2020;106(13):985-91.

6 Nestelberger T, Wildi K, Boeddinghaus J, Twerenbold R, Reichlin T, Gimenez MR, et al. Characterization of the observe zone of the ESC 2015 high-sensitivity cardiac troponin 0 $\mathrm{h} / \mathrm{l} \mathrm{h}$-algorithm for the early diagnosis of acute myocardial infarction. Int J Cardiol. 2016;207:238-45.
7 Harrell FE Jr, Lee KL, Mark DB. Multivariable prognostic models: issues in developing models, evaluating assumptions and adequacy, and measuring and reducing errors. Stat Med. 1996;15(4):361-87.

8 Backus BE, Six AJ, Kelder JC, Bosschaert MA, Mast EG, Mosterd A, et al. A prospective validation of the HEART score for chest pain patients at the emergency department. Int J Cardiol. 2013;168(3):2153-8.

9 Six AJ, Backus BE, Kelder JC. Chest pain in the emergency room: value of the HEART score. Neth Heart J. 2008;16(6):191-6.

10 Diamond GA, Forrester JS. Analysis of probability as an aid in the clinical diagnosis of coronary-artery disease. N Engl J Med. 1979; 300:1350-58.

11 Ibanez B, James S, Agewall S, Antunes MJ, Bucciarelli-Ducci C, Bueno H, et al. 2017 ESC Guidelines for the management of acute myocardial infarction in patients presenting with ST-segment elevation: The Task Force for the management of acute myocardial infarction in patients presenting with ST-segment elevation of the European Society of Cardiology (ESC). Eur Heart J. 2018;39(2):119-77.

12 Twerenbold R, Neumann JT, Sörensen NA, Ojeda F, Karakas M, Boeddinghaus J, et al. Prospective validation of the $0 / 1-\mathrm{h}$ algorithm for early diagnosis of myocardial infarction. J Am Coll Cardiol. 2018;72(6):620-32.

13 Hoffmann U, Truong QA, Schoenfeld DA, Chou ET, Woodard PK, Nagurney JT, et al. Coronary CT angiography versus standard evaluation in acute chest pain. N Engl J Med. 2012;367(4):299-308.
14 Dedic A, Lubbers MM, Schaap J, Lammers J, Lamfers EJ, Rensing BJ, et al. Coronary CT angiography for suspected ACS in the era of high-sensitivity troponins: randomized multicenter study. J Am Coll Cardiol. 2016;67(1): 16-26.

15 Arslan M, Schaap J, Van Gorsel B, Budde RP, Bekkers SC, Van Cauteren YJ, et al. Coronary $\mathrm{CT}$ angiography for improved assessment of patients with acute chest pain and low-range positive high-sensitivity troponins: study protocol for a prospective, observational, multicentre study (COURSE trial). BMJ Open. 2021;11(10):e049349.

16 Pfortmueller CA, Funk GC, Marti G, Leichtle $A B$, Fiedler GM, Schwarz C, et al. Diagnostic performance of high-sensitive troponin $\mathrm{T}$ in patients with renal insufficiency. Am J Cardiol. 2013;112(12):1968-72.

17 Twerenbold R, Wildi K, Jaeger C, Gimenez MR, Reiter M, Reichlin T, et al. Optimal cutoff levels of more sensitive cardiac troponin assays for the early diagnosis of myocardial infarction in patients with renal dysfunction. Circulation. 2015;131(23):2041-50.

18 Gunsolus I, Sandoval Y, Smith SW, Sexter A, Schulz K, Herzog CA, et al. Renal dysfunction influences the diagnostic and prognostic performance of high-sensitivity cardiac troponin I. J Am Soc Nephrol. 2018;29(2):636-43.

19 Miller-Hodges E, Anand A, Shah ASV, Chapman AR, Gallacher P, Lee KK, et al. High-sensitivity cardiac troponin and the risk stratification of patients with renal impairment presenting with suspected acute coronary syndrome. Circulation. 2018;137(5):425-35.

20 Twerenbold R, Badertscher P, Boeddinghaus J, Nestelberger T, Wildi K, Puelacher C, et al. 0/1-h triage algorithm for myocardial infarction in patients with renal dysfunction. Circulation. 2018;137(5):436-51. 